\title{
INFINITE-DIMENSIONAL SYLVESTER EQUATIONS: BASIC THEORY AND APPLICATION TO OBSERVER DESIGN
}

\author{
ZBIGNIEW EMIRSAJŁOW \\ Department of Control and Measurements \\ West Pomeranian University of Technology, Gen. Sikorskiego 37, 70-313 Szczecin, Poland \\ e-mail:emirsaj@zut.edu.pl
}

\begin{abstract}
This paper develops a mathematical framework for the infinite-dimensional Sylvester equation both in the differential and the algebraic form. It uses the implemented semigroup concept as the main mathematical tool. This concept may be found in the literature on evolution equations occurring in mathematics and physics and is rather unknown in systems and control theories. But it is just systems and control theory where Sylvester equations widely appear, and for this reason we intend to give a mathematically rigorous introduction to the subject which is tailored to researchers and postgraduate students working on systems and control. This goal motivates the assumptions under which the results are developed. As an important example of applications we study the problem of designing an asymptotic state observer for a linear infinitedimensional control system with a bounded input operator and an unbounded output operator.
\end{abstract}

Keywords: infinite-dimensional Sylvester equation, implemented semigroup, state observer design.

\section{Introduction}

As a motivation for the problem intended to be studied in this paper we provide the following finite-dimensional example of an observer design based on the algebraic Sylvester equation: Let us consider a finite-dimensional control system described by the state space model

$$
\begin{aligned}
\dot{x}(t) & =E x(t)+B u(t), \quad x(0)=x_{0}, \\
y(t) & =C x(t),
\end{aligned}
$$

where $(x(t))_{t \geq 0} \subset \mathbb{R}^{n}$ is the state trajectory, $(u(t))_{t \geq 0} \subset$ $\mathbb{R}^{m}$ is the control, $(y(t))_{t \geq 0} \subset \mathbb{R}^{p}$ is the output, $E \in$ $\mathbb{R}^{n \times n}, B \in \mathbb{R}^{n \times m}$ and $C \in \mathbb{R}^{p \times n}$ are system matrices.

For the system (1) we can consider the following asymptotic state observer:

$$
\dot{z}(t)=A z(t)+G y(t)+H u(t), \quad t \geq 0, \quad z(0)=z_{0},
$$

where $A \in \mathbb{R}^{n \times n}, G \in \mathbb{R}^{n \times p}, H \in \mathbb{R}^{n \times m}$. One can show that under suitable assumptions there exists a matrix $M \in \mathbb{R}^{n \times n}$ such that

$$
\lim _{t \rightarrow \infty}\|z(t)-M x(t)\|_{\mathbb{R}^{n}}=0 .
$$

If, additionally, this matrix is non-singular, then

$$
\lim _{t \rightarrow \infty}\left\|M^{-1} z(t)-x(t)\right\|_{\mathbb{R}^{n}}=0 .
$$

Let us notice that differentiation of the observation error $e(t)=z(t)-M x(t)$ followed by substitution of (1) and (2) leads to the differential equation:

$$
\begin{aligned}
\dot{e}(t)= & A e(t)+(A M-M E+G C) x(t) \\
& +(H-M B) u(t), \quad e(0)=z_{0}-M x_{0} .
\end{aligned}
$$

From this relation it follows that if there exists a matrix $M \in \mathbb{R}^{n \times n}$ satisfying the algebraic Sylvester equation

$$
A M-M E=-G C
$$

and the condition $H=M B$, then the observation error satisfies the homogeneous differential equation

$$
\dot{e}(t)=A e(t), \quad e(0)=z_{0}-M x_{0} .
$$

It is known (Chen, 1984) that, if the matrix $A$ is asymptotically stable and has no common eigenvalues with the matrix $E$, then the matrix equation (6) has a unique solution $M \in \mathbb{R}^{n \times n}$ and (3) holds. If, additionally, the pair $\{-E, C\}$ is observable, then we can choose a controllable pair $\{A, G\}$ such that the solution matrix $M \in \mathbb{R}^{n \times n}$ is non-singular. In this case also the condition (4) holds. 
The main goal of this paper is to extend the above approach to the infinite-dimensional case in which the control system is described by a state space model of the form (1), with $E$ and $C$ being unbounded operators, and $B$ being a bounded operator. It turns out that in this case the situation becomes extremely complicated and requires the development of a suitable mathematical framework within which the problem can be solved. For this purpose it is useful to introduce the implemented semigroup concept (see, e.g., Alber, 2001; Emirsajłow and Townley, 2005; Phong, 1991) since it is a natural tool to study the infinite-dimensional algebraic Sylvester equation. In fact, we will start our considerations with the differential Sylvester equation of the form

$$
\dot{Z}(t)=A Z(t)+Z(t) E+F(t), \quad t \geq 0, \quad Z(0)=Z_{0},
$$

where $(Z(t))_{t \geq 0}, A, E$ and $(F(t))_{t \geq 0}$ are linear operators acting in infinite-dimensional Hilbert spaces. The reason is that this equation is closely related to the algebraic Sylvester equation

$$
A Z+Z E+F=0
$$

where the solutions $Z$ and $F$ are time-invariant.

The paper is divided into five sections. Section 1 is a short introduction and Section 2 presents the main results on implemented semigroups. Then in Section 3 we provide all necessary results on infinite-dimensional Sylvester equations in both the differential and the algebraic forms. The main example illustrating the applicability of the general results to the observer design problem is described in Section 4. The paper is concluded with Section 5 containing some final remarks.

\section{Implemented semigroup}

In this section we introduce and characterize the notion of an implemented semigroup (Alber, 2001; Emirsajłow and Townley, 2005) as the main tool in the analysis of infinite-dimensional Sylvester equations. In order to state our results, we need to introduce the following notation and assumptions:

- $H^{A}$ and $H^{E}$ are Hilbert spaces (identified with their duals) with scalar products $\langle\cdot, \cdot\rangle^{A}$ and $\langle\cdot, \cdot\rangle^{E}$, respectively.

- $\mathcal{H}:=\mathscr{L}\left(H^{E}, H^{A}\right)$ is a Banach space of bounded linear operators from $H^{E}$ into $H^{A}$ with the norm $\|\cdot\| .(\mathcal{H},\|\cdot\|)$ stands for $\mathscr{L}\left(H^{E}, H^{A}\right)$ equipped with the uniform operator topology (induced by $\|\cdot\|$ ) and $(\mathcal{H}, \tau)$ stands for $\mathscr{L}\left(H^{E}, H^{A}\right)$ equipped with the strong operator topology $\tau$, i.e., the topology induced by the family of seminorms $\mathcal{P}=\left\{p_{h}\right\}_{h \in B_{1}\left(H^{E}\right)}$, where $p_{h}(X)=\|X h\|^{A}$ for $X \in \mathcal{H}$ and $h \in H^{E}$, and $B_{1}\left(H^{E}\right)$ is an open unit ball in $H^{E}$.
- $A$ is a linear, unbounded operator on $H^{A}$ generating a strongly continuous semigroup of operators $(T(t))_{t>0} \subset \mathscr{L}\left(H^{A}\right) . \quad H_{1}^{A}=\mathscr{D}(A)$ is a Hilbert space with the scalar product $\langle\cdot, \cdot\rangle_{1}^{A}=\langle(\lambda I-$ $A)(\cdot),(\lambda I-A)(\cdot)\rangle^{A}$ and the norm $\|\cdot\|_{1}^{A}$, where $\lambda \in \rho(A)$.

- $E$ is an unbounded linear operator on $H^{E}$ generating a strongly continuous semigroup of operators $(S(t))_{t \geq 0} \subset \mathscr{L}\left(H^{E}\right)$. As above, we define $H_{1}^{E}=$ $\mathscr{D}(E)$.

- $H_{-1}^{A}$ is the completion of $H^{A}$ in the norm $\|\cdot\|_{-1}^{A}=$ $\left\|(\lambda I-A)^{-1}(\cdot)\right\|^{A}$ induced by the scalar product $\langle\cdot, \cdot\rangle_{-1}^{A}=\left\langle(\lambda I-A)^{-1}(\cdot),(\lambda I-A)^{-1}(\cdot)\right\rangle^{A}$, where $\lambda \in \rho(A)$.

- $H_{-1}^{E}$ is the completion of $H^{E}$ in the norm $\|\cdot\|_{-1}^{E}=$ $\left\|(\lambda I-E)^{-1}(\cdot)\right\|^{E}$ induced by the scalar product $\langle\cdot, \cdot\rangle_{-1}^{E}=\left\langle(\lambda I-E)^{-1}(\cdot),(\lambda I-E)^{-1}(\cdot)\right\rangle^{E}$, where $\lambda \in \rho(E)$.

- $(T(t))_{t \geq 0} \subset \mathscr{L}\left(H^{A}\right)$ restricts to a strongly continuous semigroup $\left(T_{1}(t)\right)_{t \geq 0} \subset \mathscr{L}\left(H_{1}^{A}\right)$ and its generator $\left(A_{1}, \mathscr{D}\left(A_{1}\right)\right)$ is the part of $A$ in $H_{1}^{A} \subset$ $H^{A}$. Moreover, $(T(t))_{t \geq 0} \subset \mathscr{L}\left(H^{A}\right)$ extends to a strongly continuous semigroup $\left(T_{-1}(t)\right)_{t \geq 0} \subset$ $\mathscr{L}\left(H_{-1}^{A}\right)$ with generator $\left(A_{-1}, \mathscr{D}\left(A_{-1}\right)\right)$, an extension of $A$, where $\mathscr{D}\left(A_{-1}\right)=H^{A}$. Analogously, we introduce $\left(S_{1}(t)\right)_{t \geq 0} \subset \mathscr{L}\left(H_{1}^{E}\right)$ with generator $\left(E_{1}, \mathscr{D}\left(E_{1}\right)\right)$ and $\left(S_{-1}(t)\right)_{t \geq 0} \subset \mathscr{L}\left(H_{-1}^{E}\right)$ with generator $\left(E_{-1}, \mathscr{D}\left(E_{-1}\right)\right)$, where $\mathscr{D}\left(E_{-1}\right)=H^{E}$.

- $\mathcal{H}^{\sim}:=\mathscr{L}\left(H_{1}^{E}, H_{-1}^{A}\right)$ is a Banach space of bounded linear operators from $H_{1}^{E}$ into $H_{-1}^{A}$ with the norm $\|$. $\| \sim$. $\left(\mathcal{H}^{\sim},\|\cdot\| \sim\right)$ stands for $\mathscr{L}\left(H_{1}^{E}, H_{-1}^{A}\right)$ equipped with the uniform operator topology (induced by $\left.\|\cdot\|^{\sim}\right)$ and $\left(\mathcal{H}^{\sim}, \tau^{\sim}\right)$ stands for $\mathscr{L}\left(H_{1}^{E}, H_{-1}^{A}\right)$ equipped with the strong operator topology $\tau^{\sim}$, i.e., topology induced by the family of seminorms $\mathcal{P}^{\sim}=$ $\left\{p_{h}^{\sim}\right\}_{h \in B_{1}\left(H_{1}^{E}\right)}$, where $p_{h}^{\sim}(X)=\|X h\|_{H_{-1}^{A}}$ for $X \in$ $\mathscr{L}\left(H_{1}^{E}, H_{-1}^{A}\right)$ and $h \in H_{1}^{E}$.

Using the two strongly continuous semigroups $(T(t))_{t>0} \subset \mathscr{L}\left(H^{A}\right)$ and $(S(t))_{t>0} \subset \mathscr{L}\left(H^{E}\right)$ generated by $A$ and $E$, respectively, we can define another semigroup.

Definition 1. The family $(\mathcal{U}(t))_{t \geq 0} \subset \mathscr{L}(\mathcal{H})$, defined as

$$
\mathcal{U}(t) X=T(t) X S(t), \quad X \in \mathcal{H}, \quad t \geq 0,
$$

is called the implemented semigroup.

It turns out that the family $(\mathcal{U}(t))_{t>0} \subset \mathscr{L}(\mathcal{H})$ is a semigroup and for every $X \in \mathcal{H}$ it satisfies the continuity condition $\mathcal{U}(\cdot) X \in C([0, \infty) ;(\mathcal{H}, \tau))$. Such a family is said to be strongly $\tau$-continuous. In general, this family 
is not a $C_{0}$-semigroup (strongly $\|\cdot\|$-continuous in our terminology) unless both operators $A$ and $E$ are bounded. Since in infinite-dimensional systems and control theories the really interesting case is when both $A$ and $E$ are unbounded, we confine our interest exclusively to this case. This is the reason why in order to develop a relatively nice mathematical framework we have to work with strong operator topologies.

Definition 2. The infinitesimal generator $\mathcal{A}$ of the implemented semigroup $(\mathcal{U}(t))_{t \geq 0} \subset \mathscr{L}(\mathcal{H})$ is defined as the limit

$$
\mathcal{A} X=\tau-\lim _{t \searrow 0} \frac{\mathcal{U}(t) X-X}{t}, \quad X \in \mathscr{D}(\mathcal{A}),
$$

where $\mathscr{D}(\mathcal{A}) \subset \mathcal{H}$ is the domain of $\mathcal{A}$ defined as

$$
\mathscr{D}(\mathcal{A})=\left\{X \in \mathcal{H}: \tau-\lim _{t \searrow 0} \frac{\mathcal{U}(t) X-X}{t} \text { exists }\right\} .
$$

In order to get more understanding what the domain $\mathscr{D}(\mathcal{A}) \in \mathcal{H}$ and the generator $\mathcal{A}$ look like, we provide the following results:

(a) $X \in \mathcal{H}$ belongs to $\mathscr{D}(\mathcal{A})$ if and only if the restriction of $X$ to $H_{1}^{E}$ belongs to $\mathscr{L}\left(H_{1}^{E}, H_{1}^{A}\right)$ and an extension of $(A X+X E) \in \mathscr{L}\left(H_{1}^{E}, H^{A}\right)$ to $H^{E}$ belongs to $\mathcal{H}$.

(b) $\mathcal{A}$ has the following explicit representation:

$$
\begin{gathered}
(\mathcal{A} X) h=A X h+X E h, \\
X \in \mathscr{D}(\mathcal{A}), \quad h \in H_{1}^{E},
\end{gathered}
$$

where by (a) the right-hand side of this equality is well-defined in $H^{A}$.

The basic properties of the implemented semigroup can be summarized as follows:

(c) If $X \in \mathscr{D}(\mathcal{A})$, then $(\mathcal{U}(t) X)_{t \geq 0} \subset \mathscr{D}(\mathcal{A})$ and it is $\tau$-continuously differentiable in $t$, i.e., $\mathcal{U}(\cdot) X \in$ $C^{1}([0, \infty) ;(\mathcal{H}, \tau))$, and

$$
\frac{\mathrm{d}}{\mathrm{d} t} \mathcal{U}(t) X=\mathcal{A}(\mathcal{U}(t) X)=\mathcal{U}(t)(\mathcal{A} X), \quad t \geq 0 .
$$

(d) The domain $\mathscr{D}(\mathcal{A})$ is bi-dense in $\mathcal{H}$, which means that for every $X \in \mathcal{H}$ there exists a $\|\cdot\|$-bounded sequence $\left(X_{n}\right)_{n \in \mathbb{N}} \subset \mathscr{D}(\mathcal{A})$ which is convergent to $X$ in $(\mathcal{H}, \tau)$. It should be emphasized that, in general, $\mathscr{D}(\mathcal{A})$ is not dense in $(\mathcal{H},\|\cdot\|)$.

(e) The operator $(\mathcal{A}, \mathscr{D}(\mathcal{A}))$ is bi-closed, which means that for all sequences $\left(X_{n}\right)_{n \in \mathbb{N}} \subset \mathscr{D}(\mathcal{A})$ such that $\left(X_{n}\right)_{n \in \mathbb{N}}$ is $\|\cdot\|$-bounded and $\tau$ - $\lim _{n \rightarrow \infty} X_{n}=$ $X \in \mathcal{H}$ and $\left(\mathcal{A} X_{n}\right)_{n \in \mathbb{N}}$ is $\|\cdot\|$-bounded and $\tau-\lim _{n \rightarrow \infty} \mathcal{A} X_{n}=Y \in \mathcal{H}$ we have $X \in \mathscr{D}(\mathcal{A})$ and $Y=\mathcal{A} X$. (f) The following equality holds:

$$
\|\mathcal{U}(t)\|_{\mathscr{L}(\mathcal{H})}=\|T(t)\|^{A}\|S(t)\|^{E}
$$

where $t \geq 0$, and, if $\omega_{0}(T)$ is the growth bound of $(T(t))_{t \geq 0} \subset \mathscr{L}\left(X^{A}\right), \omega_{0}(S)$ is the growth bound of $(S(t))_{t \geq 0} \subset \mathscr{L}\left(X^{E}\right)$ and $\omega_{0}(\mathcal{U})$ is the growth bound of $(\overline{\mathcal{U}}(t))_{t \geq 0} \subset \mathscr{L}(\mathcal{H})$, then

$$
\omega_{0}(\mathcal{U})=\omega_{0}(T)+\omega_{0}(S) .
$$

(g) The following inclusion holds:

$$
\mathbb{C}_{\omega_{0}(T)+\omega_{0}(S)} \subset \rho(\mathcal{A}),
$$

where we use the notation

$$
\mathbb{C}_{\omega}:=\{\lambda \in \mathbb{C}: \operatorname{Re} \lambda>\omega\},
$$

with $\rho(\mathcal{A})$ denoting the resolvent set of $\mathcal{A}$, while for $\lambda \in \mathbb{C}_{\omega_{0}(T)+\omega_{0}(S)}$ the resolvent is given by

$$
\begin{aligned}
\mathcal{R}(\lambda, \mathcal{A}) X & :=(\lambda I-\mathcal{A})^{-1} X \\
& =\int_{0}^{\infty} e^{-\lambda t} \mathcal{U}(t) X \mathrm{~d} t \\
& =\int_{0}^{\infty} e^{-\lambda t} T(t) X S(t) \mathrm{d} t, \quad X \in \mathcal{H},
\end{aligned}
$$

where the integrals are convergent in $(\mathcal{H}, \tau)$.

- Throughout the rest of the paper we assume that $\lambda \in$ $\mathbb{C}_{\omega_{0}(T)+\omega_{0}(S)}$. This condition is always satisfied for sufficiently large $\lambda \in \mathbb{R}$.

- $\mathcal{H}_{1}=\mathscr{D}(\mathcal{A})$ denotes the Banach space with the norm

$$
\|X\|_{1}=\|(\lambda \mathcal{I}-\mathcal{A}) X\|, \quad X \in \mathscr{D}(\mathcal{A}) .
$$

In $\mathcal{H}_{1}$ we distinguish the uniform operator topology induced by the norm $\|\cdot\|_{1}$ and the strong operator topology $\tau_{1}$ induced by a family of seminorms $\mathcal{P}_{1}=$ $\left\{p_{1 h}\right\}_{h \in B_{1}\left(H^{E}\right)}$, where

$$
p_{1 h}(X)=p_{h}((\lambda \mathcal{I}-\mathcal{A}) X)=\|(\lambda \mathcal{I}-\mathcal{A}) X h\|^{A}
$$

for $X \in \mathscr{D}(\mathcal{A})$ and $h \in H^{E}$.

- On $\mathcal{H}$ we define another norm

$$
\|X\|_{\star}:=\|\mathcal{R}(\lambda, \mathcal{A}) X\|
$$

for $X \in \mathcal{H}$ and another family of seminorms $\mathcal{P}_{\star}=$ $\left\{p_{\star h}\right\}_{h \in B_{1}\left(H^{E}\right)}$, where

$$
p_{\star h}(X)=p_{h}(\mathcal{R}(\lambda, \mathcal{A}) X)=\|(\mathcal{R}(\lambda, \mathcal{A}) X) h\|^{A}
$$

for $X \in \mathcal{H}$ and $h \in H^{E}$, and we let $\tau_{\star}$ denote the topology on $\mathcal{H}$ induced by the family $\mathcal{P}_{\star}$. 
- $\mathcal{H}_{-1}$ is a Banach space defined as the completion of $\mathcal{H}$ understood in the sense of equivalence classes of $\|\cdot\|_{\star}$-bounded and $\tau_{\star}$-Cauchy sequences of $\mathcal{H}$. The norm on $\mathcal{H}_{-1}$ is defined by

$$
\|X\|_{-1}=\sup _{p_{-1} \in \mathcal{P}_{-1}} p_{-1 h}(X), \quad X \in \mathcal{H}_{-1},
$$

where $\mathcal{P}_{-1}=\left\{p_{-1 h}\right\}_{h \in B_{1}\left(H^{E}\right)}$ is a family of seminorms $p_{-1 h}$ on $\mathcal{H}_{-1}$ defined by the limit

$$
p_{-1 h}(X)=\lim _{n \rightarrow \infty} p_{\star h}\left(X_{n}\right), \quad h \in H^{E},
$$

$\left(X_{n}\right)_{n \in \mathbb{N}} \subset \mathcal{H}$ is any $\|\cdot\|_{\star}$-bounded and $\tau_{\star}$-Cauchy sequence from the equivalence class $X$. In $\mathcal{H}_{-1}$ we distinguish the uniform operator topology induced by the norm $\|\cdot\|_{-1}$ and the strong operator topology $\tau_{-1}$ induced by a family of seminorms $\mathcal{P}_{-1}=\left\{p_{-1 h}\right\}$, where $h \in H^{E} \cdot\left(\mathcal{H}_{-1},\|\cdot\|_{-1}\right)$ stands for $\mathcal{H}_{-1}$ equipped with the uniform topology and $\left(\mathcal{H}_{-1}, \tau_{-1}\right)$ stands for $\mathcal{H}_{-1}$ equipped with the topology $\tau_{-1}$.

One can prove the following useful relations between spaces $\mathcal{H}_{-1}$ and $\mathcal{H}$ :

(h) The topological space $\left(\mathcal{H}_{-1}, \tau_{-1}\right)$ is sequentially complete on $\|\cdot\|_{-1}$-bounded sets, and the canonical injection

$$
(\mathcal{H}, \tau) \hookrightarrow\left(\mathcal{H}_{-1}, \tau_{-1}\right)
$$

is bi-continuous and bi-dense, where bi-continuity means that every $\|\cdot\|$-bounded sequence $\left(Z_{n}\right)_{n \in \mathbb{N}} \subset$ $\mathcal{H}$ such that $\tau$ - $\lim _{n \rightarrow \infty} Z_{n}=Z \in \mathcal{H}$ is $\|\cdot\|_{-1}$-bounded and $\tau_{-1}-\lim _{n \rightarrow \infty} Z_{n}=Z$, and bidenseness means that for every $Z \in \mathcal{H}_{-1}$ there exists a $\|\cdot\|_{-1}$-bounded sequence $\left(Z_{n}\right)_{n \in \mathbb{N}} \subset \mathcal{H}$ which is $\tau_{-1}$-convergent to $Z$.

We can now define a natural extension of the implemented semigroup. This extension will play a fundamental role in our further deliberations.

- $\left(\mathcal{U}_{-1}(t)\right)_{t \geq 0} \subset \mathscr{L}\left(\mathcal{H}_{-1}\right)$ is a $\tau_{-1}$-continuous semigroup defined as follows:

$$
\begin{aligned}
\mathcal{U}_{-1}(t) X & :=\tau_{-1^{-}} \lim _{n \rightarrow \infty} \mathcal{U}(t) X_{n} \\
& =\tau_{-1^{-}} \lim _{n \rightarrow \infty} T(t) X_{n} S(t), \quad X \in \mathcal{H}_{-1},
\end{aligned}
$$

where $\left(X_{n}\right)_{n \in \mathbb{N}} \subset \mathcal{H}$ is $\|\cdot\|_{-1}$-bounded and convergent to $X$ in $\left(\mathcal{H}_{-1}, \tau_{-1}\right)$. Its generator $\left(\mathcal{A}_{-1}, \mathscr{D}\left(\mathcal{A}_{-1}\right)\right)$ (cf. Definition 2) has the domain $\mathscr{D}\left(\mathcal{A}_{-1}\right)=\mathcal{H}$ and satisfies the condition

$$
\mathcal{A}_{-1} X=\mathcal{A} X=A X+X E, \quad X \in \mathscr{D}(\mathcal{A}) .
$$

The resolvent $\mathcal{R}\left(\lambda, \mathcal{A}_{-1}\right) \in \mathscr{L}\left(\mathcal{H}_{-1}\right)$ satisfies the condition $\mathcal{R}\left(\lambda, \mathcal{A}_{-1}\right) X=\mathcal{R}(\lambda, \mathcal{A}) X$ for $X \in \mathcal{H}$. Moreover, we have

$$
\omega_{0}\left(\mathcal{U}_{-1}\right)=\omega_{0}(\mathcal{U})=\omega_{0}(T)+\omega_{0}(S) .
$$

The strongly $\tau_{-1}$-continuous semigroup $\left(\mathcal{U}_{-1}(t)\right)_{t \geq 0} \subset \mathscr{L}\left(\mathcal{H}_{-1}\right)$ characterized above is a natural extension of the implemented semigroup $(\mathcal{U}(t))_{t \geq 0} \subset \mathscr{L}(\mathcal{H})$. Unfortunately, explicit representations of $\mathcal{A}_{-1}$ and $\left(\mathcal{U}_{-1}(t)\right)_{t \geq 0}$ in terms of $A, E,(T(t))_{t \geq 0}$ and $(S(t))_{t \geq 0}$ (or their extrapolations) are not immediately obvious and require some justification. In order to deal with this problem, we explicitly define an implemented semigroup $\left(\mathcal{U}^{\sim}(t)\right)_{t>0} \subset \mathscr{L}\left(\mathcal{H}^{\sim}\right)$ whose restriction to $\mathcal{H}_{-1}$ coincides with $\left(\mathcal{U}_{-1}(t)\right)_{t \geq 0} \subset \mathscr{L}\left(\mathcal{H}_{-1}\right)$

- $\left(\mathcal{U}^{\sim}(t)\right)_{t \geq 0} \subset \mathscr{L}\left(\mathcal{H}^{\sim}\right)$ is an implemented semigroup on $\mathscr{L}\left(\mathcal{H}^{\sim}\right)$ defined as follows:

$$
\mathcal{U}^{\sim}(t) X:=T_{-1}(t) X S_{1}(t), \quad X \in \mathcal{H}^{\sim} .
$$

It is clear that $\left(\mathcal{U}^{\sim}(t)\right)_{t \geq 0} \subset \mathscr{L}\left(\mathcal{H}^{\sim}\right)$ and it is strongly $\tau^{\sim}$-continuous in time. By $\left(\mathcal{A}^{\sim}, \mathscr{D}\left(\mathcal{A}^{\sim}\right)\right)$ we denote its generator with the domain $\mathscr{D}\left(\mathcal{A}^{\sim}\right)$.

The following results hold:

(i) $X \in \mathscr{D}\left(\mathcal{A}^{\sim}\right)$ if and only if the restriction of $X$ to $H_{2}^{E}=\mathscr{D}\left(E_{1}\right)$ belongs to $\mathscr{L}\left(H_{2}^{E}, H^{A}\right)$, i.e., $\mathscr{D}\left(\mathcal{A}^{\sim}\right) \subset \mathcal{H}^{\sim} \cap \mathscr{L}\left(H_{2}^{E}, H^{A}\right)$, and the extension of $\left(A_{-1} X+X E_{1}\right) \in \mathscr{L}\left(H_{2}^{E}, H_{-1}^{A}\right)$ to $H_{1}^{E}$ belongs to $\mathcal{H}^{\sim}$. Moreover, operator $\mathcal{A}^{\sim}$ admits the following explicit representation:

$$
\left(\mathcal{A}^{\sim} X\right) h=A_{-1} X h+X E_{1} h,
$$

where $\quad X \in \mathscr{D}\left(\mathcal{A}^{\sim}\right), h \in H_{2}^{E}$, and the right-hand side of (21) is well-defined in $H_{-1}^{A}$.

(j) We have

$$
\begin{aligned}
\omega_{0}\left(\mathcal{U}^{\sim}\right) & =\omega_{0}\left(T_{-1}\right)+\omega_{0}\left(S_{1}\right)=\omega_{0}(T)+\omega_{0}(S) \\
& =\omega_{0}(\mathcal{U})
\end{aligned}
$$

and for $\lambda \in \mathbb{C}_{\omega_{0}(T)+\omega_{0}(S)}$

$$
\begin{aligned}
\mathcal{R}\left(\lambda, \mathcal{A}^{\sim}\right) X & =\int_{0}^{\infty} e^{-\lambda t} \mathcal{U}^{\sim}(t) X \mathrm{~d} t \\
& =\int_{0}^{\infty} e^{-\lambda t} T_{-1}(t) X S_{1}(t) \mathrm{d} t,
\end{aligned}
$$

where $X \in \mathcal{H}^{\sim}$ and the integrals are convergent in $\left(\mathcal{H}^{\sim}, \tau^{\sim}\right)$.

(k) The canonical injection

$$
(\mathcal{H}, \tau) \hookrightarrow\left(\mathcal{H}^{\sim}, \tau^{\sim}\right)
$$

is bi-continuous and bi-dense, where bi-continuity means that every $\|\cdot\|$-bounded sequence $\left(Z_{n}\right)_{n \in \mathbb{N}} \subset$ $\mathcal{H}$ such that $\tau$ - $\lim _{n \rightarrow \infty} Z_{n}=Z \in \mathcal{H}$ is $\|\cdot\|^{\sim}$-bounded and $\tau^{\sim} \lim _{n \rightarrow \infty} Z_{n}=Z$, and bidenseness means that for every $Z \in \mathcal{H}^{\sim}$ there exists a $\|\cdot\|^{\sim}$-bounded sequence $\left(Z_{n}\right)_{n \in \mathbb{N}} \subset \mathcal{H}$ which is $\tau^{\sim}$-convergent to $Z$. 
(1) We have $\mathcal{H} \subset \mathscr{D}\left(\mathcal{A}^{\sim}\right)$, and $\mathcal{A}^{\sim}$ admits the following explicit representation

$$
\left(\mathcal{A}^{\sim} X\right) h=A_{-1} X h+X E h,
$$

where $X \in \mathcal{H}, h \in H_{1}^{E}$, and equality holds in $H_{-1}^{A}$.

(m) Every $X \in \mathcal{H}_{-1}$ can be uniquely identified with an element in $\mathcal{H}^{\sim}=\mathscr{L}\left(H_{1}^{E}, H_{-1}^{A}\right)$, i.e., we have $\mathcal{H}_{-1} \subset \mathcal{H}^{\sim}$, and the canonical injection

$$
\left(\mathcal{H}_{-1}, \tau_{-1}\right) \hookrightarrow\left(\mathcal{H}^{\sim}, \tau^{\sim}\right)
$$

is bi-continuous and bi-dense, where bi-continuity means that every $\|\cdot\|_{-1}$-bounded sequence $\left(Z_{n}\right)_{n \in \mathbb{N}} \subset \mathcal{H}_{-1}$ such that $\tau_{-1}-\lim _{n \rightarrow \infty} Z_{n}=Z \in$ $\mathcal{H}_{-1}$ is $\|\cdot\|^{\sim}$-bounded and $\tau^{\sim}-\lim _{n \rightarrow \infty} Z_{n}=Z$, and bi-denseness means that for every $Z \in \mathcal{H}^{\sim}$ there exists a $\|\cdot\|^{\sim}$-bounded sequence $\left(Z_{n}\right)_{n \in \mathbb{N}} \subset \mathcal{H}_{-1}$ which is $\tau^{\sim}$-convergent to $Z$.

(n) The following relations hold:

$$
\begin{aligned}
\left(\mathcal{A}_{-1} X\right) h & =\left(\mathcal{A}^{\sim} X\right) h \\
& =A_{-1} X h+X E h,
\end{aligned}
$$

where $X \in \mathcal{H}, h \in H_{1}^{E}$,

$$
\begin{aligned}
\left(\mathcal{U}_{-1}(t) X\right) h & =\left(\mathcal{U}^{\sim}(t) X\right) h \\
& =T_{-1}(t) X S_{1}(t) h,
\end{aligned}
$$

where $X \in \mathcal{H}_{-1}, t \geq 0, h \in H_{1}^{E}$,

$$
\begin{aligned}
\left(\mathcal{R}\left(\lambda, \mathcal{A}_{-1}\right) X\right) h & =\left(\mathcal{R}\left(\lambda, \mathcal{A}^{\sim}\right) X\right) h \\
& =\int_{0}^{\infty} e^{-\lambda t} T_{-1}(t) X S_{1}(t) h \mathrm{~d} t
\end{aligned}
$$

where $X \in \mathcal{H}_{-1}, h \in H_{1}^{E}$, and equalities hold in $H_{-1}^{A}$.

\section{Infinite-dimensional Sylvester equations}

In this section we examine infinite-dimensional Sylvester equations which are in a natural way related to the implemented semigroup. The algebraic Sylvester equation will play a crucial role in the observer design problem analyzed in the next section. Throughout this section we assume that

$$
F \in \mathcal{H}^{\sim}=\mathscr{L}\left(H_{1}^{E}, H_{-1}^{A}\right)
$$

3.1. Differential Sylvester equation. From (13) it immediately follows that, if $Z_{0} \in \mathcal{H}_{1}$, then the expression

$$
Z(t)=\mathcal{U}(t) Z_{0}=T(t) Z_{0} S(t), \quad t \geq 0,
$$

satisfies the following conditions:

$$
Z(t) \in \mathcal{H}_{1}, \quad \dot{Z}(t)=\mathcal{A}\left(\mathcal{U}(t) Z_{0}\right) \in \mathcal{H},
$$

where $t \geq 0, \quad Z(0)=Z_{0}$, which show that $(Z(t))_{t \geq 0}$ can be viewed as a solution to the initial value problem (26). However, the differentiation in (26) is understood in $(\mathcal{H}, \tau)$ and, in general, does not make sense in $(\mathcal{H},\|\cdot\|)$. This means that the notion of a classical strong solution is inappropriate here since it would require the function $(Z(t))_{t \geq 0}$ to satisfy

$$
Z(\cdot) \in C^{1}([0, \infty) ;(\mathcal{H},\|\cdot\|)) .
$$

Nevertheless, the expression 25 can still be regarded as a strong solution of the initial value problem

$$
\dot{Z}(t)=\mathcal{A} Z(t), \quad t \geq 0, \quad Z(0)=Z_{0},
$$

but we have to consider this problem on $(\mathcal{H}, \tau)$ rather than on $(\mathcal{H},\|\cdot\|)$. As usual we will refer to (27) as the homogeneous Cauchy problem and, since we have $(Z(t))_{t \geq 0} \subset \mathcal{H}_{1}$, then according to basic properties of the implemented semigroup, we can rewrite the differential equation 27 in the more explicit form

$$
\dot{Z}(t) h=A Z(t) h+Z(t) E h, \quad t \geq 0,
$$

where $Z(0)=Z_{0}, h \in H_{1}^{E}$, and the equality holds in $H^{A}$. In this case we will refer to the differential equation (28) as the homogeneous differential Sylvester equation.

However, the main aim of this section is to study the infinite-dimensional differential Sylvester equation with an unbounded input, i.e.,

$$
\begin{gathered}
\dot{Z}(t)=A_{-1} Z(t)+Z(t) E+F(t), \\
t \geq 0, \quad Z(0)=Z_{0}
\end{gathered}
$$

where $Z_{0} \in \mathcal{H}, F(\cdot) \in C\left([0, \infty) ;\left(\mathcal{H}^{\sim}, \tau^{\sim}\right)\right)$. In particular, we study in detail the important case when the input is time-invariant, i.e., $F \in \mathcal{H}^{\sim}$, and for such inputs we identify the class of the so-called admissible input elements. We also use the implemented semigroup approach to study the algebraic Sylvester equation of the form

$$
\omega Z-A_{-1} Z-Z E=F,
$$

where $\omega>\omega_{0}(\mathcal{U})$ and $F \in \mathcal{H}^{\sim}$. Our study makes direct use of the extrapolation (extension) results for the implemented semigroups and is presented in the next section. Some basic results for the case with a bounded operator $F \in \mathcal{H}$ can be also found in the work of Phong (1991).

3.2. Inhomogeneous differential Sylvester equation. The first goal is to consider the inhomogeneous Cauchy problem of the form

$$
\dot{Z}(t)=\mathcal{A}^{\sim} Z(t)+F(t), \quad t \geq 0, \quad Z(0)=Z_{0},
$$

where $Z_{0} \in \mathcal{H}$ and $(F(t))_{t \geq 0} \subset \mathcal{H}^{\sim}$. Motivated by applications of 29) in infinite-dimensional systems and control theory, we are interested in solutions which satisfy 
the condition $(Z(t))_{t>0} \subset \mathcal{H}$. For an example of application of the inhomogeneous differential Lyapunov equation (Sylvester equation with $E=A^{*}$ ) in an optimal control problem, see the work of Emirsajłow and Townley (2005). From some previous results it follows that for $Z \in \mathcal{H}$ we have $\mathcal{A}_{\sim} Z=\mathcal{A}_{-1} Z$, and hence the differential equation (29) becomes

$$
\dot{Z}(t)=\mathcal{A}_{-1} Z(t)+F(t), \quad t \geq 0, \quad Z(0)=Z_{0},
$$

and can be written explicitly as

$$
\dot{Z}(t) h=A_{-1} Z(t) h+Z(t) E h+F(t) h, \quad t \geq 0,
$$

where $Z(0)=Z_{0}, h \in H_{1}^{E}$, and the equality holds in $H_{-1}^{A}$. The differential equation (31) is referred to as the inhomogeneous differential Sylvester equation with an unbounded input. The term "unbounded input" is justified by the fact that we are looking for solutions $(Z(t))_{t \geq 0}$ with values in $\mathcal{H}$ while the input function $(F(t))_{t \geq 0}$ is allowed to take values in a bigger Banach space $\mathcal{H}^{\sim}$ which contains $\mathcal{H}$.

In order to state basic results on the strong solution, we need the following additional definitions:

- $\mathcal{H}_{1}^{\sim}=\mathscr{D}\left(\mathcal{A}^{\sim}\right)$ is a Banach space endowed with the norm

$$
\|Z\|_{1}^{\sim}:=\left\|\left(\lambda I-\mathcal{A}^{\sim}\right) Z\right\|^{\sim}, \quad Z \in \mathscr{D}\left(\mathcal{A}^{\sim}\right),
$$

where $\lambda \in \mathbb{C}_{\omega_{0}}(\mathcal{U})$.

- On $\mathcal{H}_{1}^{\sim}$ we introduce a topology $\tau_{1}^{\sim}$ induced by a family of seminorms $\mathcal{P}_{1}^{\sim}=\left\{p_{1 h}^{\sim}(\cdot)\right\}_{h \in B_{1}\left(H_{1}^{E}\right)}$, where

$$
\begin{aligned}
p_{1 h}^{\sim}(Z) & :=p_{h}^{\sim}\left(\left(\lambda I-\mathcal{A}^{\sim}\right) Z\right) \\
& =\left\|\left(\left(\lambda I-\mathcal{A}^{\sim}\right) Z\right) h\right\|_{-1}^{A},
\end{aligned}
$$

$Z \in \mathcal{H}_{1}^{\sim}$ and $h \in H_{1}^{E} .\left(\mathcal{H}_{1}^{\sim},\|\cdot\|_{1}^{\sim}\right)$ stands for $\mathcal{H}_{1}^{\sim}$ endowed with the norm topology. $\left(\mathcal{H}_{1}^{\sim}, \tau_{1}^{\sim}\right)$ stands for $\mathcal{H}_{1}^{\sim}$ endowed with the topology $\tau_{1}^{\sim}$.

Definition 3. Let us consider the inhomogeneous Cauchy problem (29) on the topological space $\left(\mathcal{H}^{\sim}, \tau^{\sim}\right)$ and assume that $Z_{0} \in \mathcal{H}$ and $F(\cdot) \in C\left([0, \infty) ;\left(\mathcal{H}^{\sim}, \tau^{\sim}\right)\right)$. A function $(Z(t))_{t \geq 0}$ is said to be a strong solution of 29] if it satisfies the following conditions:

(i) $Z(\cdot) \in C^{1}\left([0, \infty) ;\left(\mathcal{H}^{\sim}, \tau^{\sim}\right)\right)$.

(ii) $(Z(t))_{t>0} \subset \mathcal{H}_{1}^{\sim}$ for $t \geq 0, Z(0)=Z_{0}$ and $\dot{Z}(t)=$ $\mathcal{A}^{\sim} Z(t)+F(t)$ holds in $\mathcal{H}^{\sim}$ for every $t \geq 0$.

It is also clear that every function $(Z(t))_{t>0}$ which is a strong solution of 29) also satisfies the condition

$$
Z(\cdot) \in C\left([0, \infty) ;\left(\mathcal{H}_{1}^{\sim}, \tau_{1}^{\sim}\right)\right) .
$$

The following theorem characterizes the strong solution of the differential equation (29). A detailed proof of this result can be found in the work of Emirsajłow (2005).
Theorem 1. Let $F(\cdot) \in C^{1}\left([0, \infty) ;\left(\mathcal{H}^{\sim}, \tau^{\sim}\right)\right)$. Then the inhomogeneous differential Sylvester equation (31) has a unique strong solution $(Z(t))_{t \geq 0}$ given by the expression

$$
\begin{aligned}
Z(t)= & \mathcal{U}^{\sim}(t) Z_{0}+\int_{0}^{t} \mathcal{U}^{\sim}(t-r) F(r) \mathrm{d} r \\
= & T_{-1}(t) Z_{0} S_{1}(t) \\
& +\int_{0}^{t} T_{-1}(t-r) F(r) S_{1}(t-r) \mathrm{d} r .
\end{aligned}
$$

However, (35) takes values in $H_{1}^{\sim}=\mathscr{D}\left(\mathcal{A}^{\sim}\right)$, which is larger than $\mathcal{H}$, and an important problem is to characterize inputs $F(\cdot) \in C\left([0, \infty) ;\left(\mathcal{H}^{\sim}, \tau^{\sim}\right)\right)$ such that for every $Z_{0} \in \mathcal{H}$ the differential Sylvester equation (31) has a solution $Z(\cdot) \in C([0, \infty) ;(\mathcal{H}, \tau))$. In general, it is a difficult and unsolved problem. However, a very useful partial solution can be obtained by restating Theorem 1 for the space $\mathcal{H}_{-1}$. Before that, we point out that every function $(F(t))_{t \geq 0}$ such that $F(\cdot) \in C^{1}([0, \infty) ;(\mathcal{H}, \tau))$, also satisfies $F(\cdot) \in C^{1}\left([0, \infty) ;\left(\mathcal{H}_{-1}, \tau_{-1}\right)\right)$, which in turn implies that $F(\cdot) \in C^{1}\left([0, \infty) ;\left(\mathcal{H}^{\sim}, \tau^{\sim}\right)\right)$.

Theorem 2. Let $F(\cdot) \in C^{1}\left([0, \infty) ;\left(\mathcal{H}_{-1}, \tau_{-1}\right)\right)$ and $Z_{0} \in \mathcal{H}$. Then the inhomogeneous differential Sylvester equation (31) has a unique strong solution $(Z(t))_{t \geq 0}$ which satisfies the condition

$$
Z(\cdot) \in C([0, \infty) ;(\mathcal{H}, \tau)) \cap C^{1}\left([0, \infty) ;\left(\mathcal{H}_{-1}, \tau_{-1}\right)\right)
$$

and can be expressed in the form

$$
\begin{aligned}
Z(t)= & \mathcal{U}_{-1}(t) Z_{0}+\int_{0}^{t} \mathcal{U}_{-1}(t-r) F(r) \mathrm{d} r \\
= & T_{-1}(t) Z_{0} S_{1}(t) \\
& +\int_{0}^{t} T_{-1}(t-r) F(r) S_{1}(t-r) \mathrm{d} r .
\end{aligned}
$$

Since in infinite-dimensional systems and control theories the most important case of the differential Sylvester equation (31) corresponds to a time-invariant input $F \in \mathcal{H}^{\sim}$, we will study this case in greater detail in the next subsection. In order to be more precise, we are interested in solutions $(Z(t))_{t \geq 0} \subset \mathcal{H}$ of the differential equation

$$
\dot{Z}(t)=\mathcal{A}^{\sim} Z(t)+F, \quad t \geq 0, \quad Z(0)=Z_{0},
$$

where $Z_{0} \in \mathcal{H}$ and $F \in \mathcal{H}^{\sim}$. From the property (23) we know that under the assumption $(Z(t))_{t \geq 0} \subset \mathcal{H}$ the differential equation (38) becomes

$$
\dot{Z}(t)=\mathcal{A}_{-1} Z(t)+F, \quad t \geq 0, \quad Z(0)=Z_{0},
$$

and can be written explicitly as the infinite-dimensional differential Sylvester equation with an unbounded input element

$$
\dot{Z}(t) h=A_{-1} Z(t) h+Z(t) E h+F h,
$$

where $h \in H_{1}^{E}, t \geq 0, Z(0)=Z_{0} \in \mathcal{H}, F \in \mathcal{H}^{\sim}$, and the equality in (40) is understood in $H_{-1}^{A}$. 
3.2.1. Admissible input elements. Since $F \in \mathcal{H}^{\sim}$, the strong solution of the differential equation (38) (or the differential Sylvester equation (40) takes values in $\mathcal{H}_{1}^{\sim}$, which is larger than $\mathcal{H}$. From the application point of view, a very important problem is to characterize all $F \in \mathcal{H}^{\sim}$ such that for every $Z_{0} \in \mathcal{H}$ Eqn. (40) has a solution $Z(\cdot) \in C([0, \infty) ;(\mathcal{H}, \tau))$. Such elements $F$ will be called admissible input elements by a close analogy with the concept of admissible control operators known in systems and control theories. For the latter concept and how these two issues are related to each other, see, e.g., the works of Emirsajłow and Townley $(2000 ; 2005)$.

Since for every $Z_{0} \in \mathcal{H}$ we have $\left(\mathcal{U}^{\sim}(t) Z_{0}\right)_{t \geq 0}=$ $\left(\mathcal{U}(t) Z_{0}\right)_{t \geq 0} \subset \mathcal{H}$, without loss of generality we can assume $Z_{0}=0$ and restrict our considerations only to the forced term of the solution. For the sake of simplicity we introduce a family $((\mathcal{M} F)(t))_{t \geq 0} \subset \mathcal{H}^{\sim}$ defined as follows:

$$
\begin{aligned}
(\mathcal{M} F)(t) & :=\int_{0}^{t} \mathcal{U}^{\sim}(t-r) F \mathrm{~d} r \\
& =\int_{0}^{t} T_{-1}(t-r) F S_{1}(t-r) \mathrm{d} r
\end{aligned}
$$

where $t \geq 0$ and $F \in \mathcal{H}^{\sim}$.

Definition 4. $F \in \mathcal{H}^{\sim}$ is said to be an admissible input element for the differential Sylvester equation (40) if there exist $\varepsilon>0$ and $C>0$ such that

$$
\begin{aligned}
(\mathcal{M} F)(t) & \in \mathcal{H}, \quad t \in[0, \varepsilon], \\
\sup _{0 \leq t \leq \varepsilon}\|(\mathcal{M} F)(t)\| & \leq C
\end{aligned}
$$

and

$$
\tau-\lim _{t \searrow 0}(\mathcal{M} F)(t)=0
$$

The above concept of the admissibility of an input element $F \in \mathcal{H}^{\sim}$ has the following consequences.

Lemma 1. If $F \in \mathcal{H}^{\sim}$ is an admissible input element for the differential equation (40), then we have

$$
(\mathcal{M F})(t) \in \mathcal{H}, \quad t \geq 0
$$

and

$$
(\mathcal{M F})(\cdot) \in C([0, \infty) ;(\mathcal{H}, \tau)) .
$$

The proof of this lemma is given in Appendix A.

Before stating the main theorem on the admissibility of input elements $F \in \mathcal{H}^{\sim}$, we need the following technical result.

Lemma 2. For $F \in \mathcal{H}^{\sim}$ and $t \geq 0$, the following identity holds in $\mathcal{H}^{\sim}$ :

$$
\begin{aligned}
t F= & \int_{0}^{t} \mathcal{U}^{\sim}(t-r) F \mathrm{~d} r-t \mathcal{A}^{\sim} \int_{0}^{t} \mathcal{U}^{\sim}(t-r) F \mathrm{~d} r \\
& +\mathcal{A}^{\sim} \int_{0}^{t} \mathcal{U}^{\sim}(t-r)\left(\int_{0}^{r} \mathcal{U}^{\sim}(r-s) F \mathrm{~d} s\right) \mathrm{d} r,
\end{aligned}
$$

where all integrals are Riemann $\tau^{\sim}$-integrals.
The proof of this lemma is given in Appendix B.

The above lemma allows us to prove the following important result characterizing all admissible elements.

Theorem 3. $F \in \mathcal{H}^{\sim}$ is an admissible input element for the differential equation (38) (equivalently, for the differential Sylvester equation (40)) if and only if

$$
F \in \mathcal{H}_{-1} \text {. }
$$

Proof.

(Sufficiency) Let $\lambda \in \rho(\mathcal{A})$ and $F \in \mathcal{H}_{-1}$. It follows from the properties of the implemented semigroup that for $t \geq 0$

$$
\begin{aligned}
& \left(\lambda I-\mathcal{A}_{-1}\right) \int_{0}^{t} \mathcal{U}_{-1}(t-r) F d r \\
& =\lambda \int_{0}^{t} \mathcal{U}_{-1}(t-r) F d r-\mathcal{U}_{-1}(t) F+F,
\end{aligned}
$$

which holds in $\mathcal{H}_{-1}$. Introducing $F_{\lambda}:=\mathcal{R}\left(\lambda, \mathcal{A}_{-1}\right) F \in$ $\mathcal{H}$ and using definition (41), we obtain the following expression for $t \geq 0$ :

$$
\begin{aligned}
(\mathcal{M} F)(t)= & \int_{0}^{t} \mathcal{U}_{-1}(t-r) F \mathrm{~d} r \\
= & \lambda \int_{0}^{t} \mathcal{U}(t-r) F_{\lambda} \mathrm{d} r \\
& -(\mathcal{U}(t)-I) F_{\lambda} .
\end{aligned}
$$

This expression shows that (42) holds. In turn, the relations (43) and (44) also easily follow from (49) if we use the basic properties of the implemented semigroup $(\mathcal{U}(t))_{t \geq 0} \subset \mathscr{L}(\mathcal{H})$. Thus, according to Definition 4, $F \in \mathcal{H}_{-1}$ is an admissible input element for the differential equation (38), or equivalently, for the differential Sylvester equation 40].

(Necessity) Let $F \in \mathcal{H}^{\sim}$ be an admissible input element. Lemma 2 for $t=1$ yields

$$
\begin{aligned}
F= & \int_{0}^{1} \mathcal{U}^{\sim}(1-r) F \mathrm{~d} r-\mathcal{A}^{\sim} \int_{0}^{1} \mathcal{U}^{\sim}(1-r) F \mathrm{~d} r \\
& +\mathcal{A}^{\sim} \int_{0}^{1} \mathcal{U}^{\sim}(1-r)\left(\int_{0}^{r} \mathcal{U}^{\sim}(r-s) F \mathrm{~d} s\right) \mathrm{d} r
\end{aligned}
$$

and hence

$$
F=(\mathcal{M F})(1)-\mathcal{A}_{-1}((\mathcal{M} F)(1))+\mathcal{A}_{-1}((\mathcal{M M} \mathcal{M} F)(1)) .
$$

Since the right-hand side of this equality belongs to $\mathcal{H}_{-1}$, so does the left-hand side, i.e., $F \in \mathcal{H}_{-1}$.

The above results lead to the following important corollary. 
Corollary 1. Let $Z_{0} \in \mathcal{H}$. The inhomogeneous differential Sylvester equation (40) has a unique strong solution

$$
Z(\cdot) \in C([0, \infty) ;(\mathcal{H}, \tau)) \cap C^{1}\left([0, \infty) ;\left(\mathcal{H}_{-1}, \tau_{-1}\right)\right)
$$

(hence also $Z(\cdot) \in C^{1}\left([0, \infty) ;\left(\mathcal{H}^{\sim}, \tau^{\sim}\right)\right)$ ) if and only if $F \in \mathcal{H}_{-1}$, and this solution can be expressed in the form

$$
\begin{aligned}
Z(t)= & \mathcal{U}(t) Z_{0}+\int_{0}^{t} \mathcal{U}_{-1}(t-r) F \mathrm{~d} r \\
= & T(t) Z_{0} S(t) \\
& +\int_{0}^{t} T_{-1}(t-r) F S_{1}(t-r) \mathrm{d} r, \quad t \geq 0 .
\end{aligned}
$$

We see that the space $\mathcal{H}_{-1}$ plays a crucial role in analysis of the differential Sylvester equation (40). Detailed analysis of this equation leads to very useful results on the algebraic Sylvester equations, which are dealt with in the next subsection.

3.3. Algebraic Sylvester equation. For algebraic Sylvester equations we have the following results.

Proposition 1. Let $\omega>\omega_{0}(\mathcal{U})=\omega_{0}(T)+\omega_{0}(S)$ and $F \in \mathcal{H}^{\sim}$. Then the algebraic equation

$$
\left(\omega I-\mathcal{A}^{\sim}\right) Z=F,
$$

understood in $\mathcal{H}^{\sim}$, or equivalently the Algebraic Sylvester Equation (ASE)

$$
\omega Z-A_{-1} Z h-Z E h=F h, \quad h \in H_{1}^{E},
$$

which holds in $H_{-1}^{A}$, has a unique solution $Z \in \mathcal{H}$ if and only if

$$
F \in \mathcal{H}_{-1},
$$

or, equivalently,

$$
\mathcal{R}\left(\omega, \mathcal{A}^{\sim}\right) F \in \mathcal{H}
$$

Moreover, if the solution $Z \in \mathcal{H}$ exists, then it is given by the expression

$$
\begin{aligned}
Z & =\mathcal{R}\left(\omega, \mathcal{A}^{\sim}\right) F \\
& =\mathcal{R}\left(\omega, \mathcal{A}_{-1}\right) F \\
& =\int_{0}^{\infty} e^{-\omega t} \mathcal{U}_{-1}(t) F \mathrm{~d} t \\
& =\int_{0}^{\infty} e^{-\omega t} T_{-1}(t) F S_{1}(t) \mathrm{d} t .
\end{aligned}
$$

Proof. Since for $\lambda \in \mathbb{C}_{\omega_{0}(\mathcal{U})}$ the operator $\left(\lambda I-\mathcal{A}_{-1}\right)$ is an isometric isomorphism from $\mathcal{H}$ onto $\mathcal{H}_{-1}$ and

$$
\left.\left(\lambda I-\mathcal{A}^{\sim}\right)\right|_{\mathcal{H}}=\left(\lambda I-\mathcal{A}_{-1}\right),
$$

it follows that the equation

$$
\left(\lambda I-\mathcal{A}^{\sim}\right) Z=F \in \mathcal{H}^{\sim}
$$

has a solution $Z \in \mathcal{H}$ if and only if $F \in \mathcal{H}_{-1}$. Moreover, this solution is always unique. Using the explicit representation (23), we can rewrite (54) in the form (51). The condition (53) follows immediately from (54) since $\mathcal{R}\left(\lambda, \mathcal{A}^{\sim}\right):=\left(\lambda I-\mathcal{A}^{\sim}\right)^{-1} \in \mathscr{L}\left(\mathcal{H}^{\sim}\right)$.

Remark 1. Proposition 1 shows that all elements $F \in$ $\mathcal{H}_{-1}$ can be parameterized by the formula

$$
F(Z)=\lambda Z-A_{-1} Z-Z E, \quad Z \in \mathcal{H},
$$

where $\lambda \in \mathbb{C}_{\omega_{0}(\mathcal{U})}$ is a fixed but arbitrary number.

In the very important case when the implemented semigroup $(\mathcal{U}(t))_{t \geq 0} \subset \mathscr{L}(\mathcal{H})$ is uniformly exponentially stable $\left(\omega_{0}(\mathcal{U})<0\right)$, we can complement Proposition 1 as follows

Corollary 2. Let $\omega_{0}(\mathcal{U})=\omega_{0}(T)+\omega_{0}(S)<0$ and $F \in$ $\mathcal{H}^{\sim}$. Then the algebraic equation

$$
-\mathcal{A}^{\sim} Z=F
$$

understood in $\mathcal{H}^{\sim}$, or equivalently the ASE

$$
-A_{-1} Z h-Z E h=F h, \quad h \in H_{1}^{E},
$$

which holds in $H_{-1}^{A}$, has a unique solution $Z \in \mathcal{H}$ if and only if $F \in \mathcal{H}_{-1}$. Moreover, if the solution $Z \in \mathcal{H}$ exists, then it is given by the expression

$$
\begin{aligned}
Z & =\left(-\mathcal{A}^{\sim}\right)^{-1} F \\
& =\left(-\mathcal{A}_{-1}\right)^{-1} F \\
& =\int_{0}^{\infty} \mathcal{U}_{-1}(t) F \mathrm{~d} t \\
& =\int_{0}^{\infty} T_{-1}(t) F S_{1}(t) \mathrm{d} t .
\end{aligned}
$$

Some useful results for the solvability of the algebraic Sylvester equation (57) with a bounded operator $F \in \mathcal{H}$ based on spectral properties of the operators $A$ and $E$ in various special cases can be found in the work of Arendt et al. (1994).

\section{Asymptotic observer design}

In order to study the infinite-dimensional version of the observer design problem mentioned in Section 1, we introduce the following additional notation and assumptions:

- The family $(S(t))_{t \in \mathbb{R}} \subset \mathscr{L}\left(H^{E}\right)=\mathcal{H}^{E}$ is a strongly continuous group with generator $(E, \mathscr{D}(E))$ and the growth bound $\omega_{0}(S)$ defined as follows:

$$
\begin{aligned}
& \omega_{0}(S) \\
& =\inf \left\{\omega \in \mathbb{R}: \quad \exists M_{\omega} \geq 1:\right. \\
& \\
& \left.\|S(t)\|_{\mathcal{H}^{E}} \leq M_{\omega} e^{\omega|t|}, \quad t \in \mathbb{R}\right\} .
\end{aligned}
$$


Consequently, the family $(S(t))_{t>0} \subset \mathcal{H}^{E}$ is a strongly continuous semigroup with generator $(E, \mathscr{D}(E))$ and growth bound $\omega_{0}(S)$ and the family $(S(-t))_{t \geq 0} \subset \mathcal{H}^{E}$ is a strongly continuous semigroup with generator $(-E, \mathscr{D}(E))$ and growth bound $\omega_{0}(S)$ (see Engel and Nagel, 2000).

- $U$ and $Y$ are additional Hilbert spaces called the output and input spaces, respectively

- $B \in \mathscr{L}\left(U, H^{E}\right)$ is a (bounded) input operator.

- $C \in \mathscr{L}\left(H_{1}^{E}, Y\right)$ is an (unbounded) output operator.

Under the above assumptions, we consider the following infinite-dimensional control system:

$$
\begin{aligned}
& \dot{x}(t)=E x(t)+B u(t), \quad x(0)=x_{0}, \\
& y(t)=C x(t),
\end{aligned}
$$

where $(x(t))_{t \geq 0}$ is the state trajectory, $(u(t))_{t \geq 0} \subset U$ is the control and $(y(t))_{t>0} \subset Y$ is the output.

For the system (61) we want to design an asymptotic state observer based on the same idea as described in Section 1 for the finite-dimensional control system. In order to do that, we consider the following infinite-dimensional dynamical system:

$$
\dot{z}(t)=A_{-1} z(t)+G y(t)+H u(t), \quad z(0)=z_{0},
$$

where $(z(t))_{t \geq 0}$ is the state trajectory, under the following assumptions:

- The family $(T(t))_{t \geq 0} \subset \mathscr{L}\left(H^{A}\right)=\mathcal{H}^{A}$ is a uniformly exponentially stable strongly continuous semigroup with generator $(A, \mathscr{D}(A))$. In this case the growth bound $\omega_{0}(T)$ is negative, i.e., $\omega_{0}(T)<0$.

- $G \in \mathscr{L}\left(Y, H_{-1}^{A}\right)$ is an (unbounded) output operator.

- $H \in \mathscr{L}\left(U, H^{A}\right)$ is a (bounded) input operator.

Let us notice that for $x_{0} \in H_{2}^{E}$ and $u(\cdot) \in$ $C^{2}([0, \infty) ; U)$ such that $u(0)=0$ the trajectory $(x(t))_{t \geq 0}$ and the corresponding output $(y(t))_{t \geq 0}$ of the system (61) enjoy the following regularity:

$$
x(\cdot) \in C^{1}\left([0, \infty) ; H_{1}^{E}\right), \quad y(\cdot) \in C^{1}([0, \infty) ; Y),
$$

and hence for $z_{0} \in H^{A}$ the trajectory $(z(t))_{t \geq 0}$ of the system (62) satisfies the condition

$$
z(\cdot) \in C\left([0, \infty) ; H^{A}\right) \cap C^{1}\left([0, \infty) ; H_{-1}^{A}\right) .
$$

In the above considerations $H_{2}^{E}$ denotes the domain of $E_{1}$ understood as an unbounded operator on $H_{1}^{E}$. The corresponding definitions of the interpolation spaces $H_{k}^{E}$ and $H_{k}^{A}$ as well as the extrapolation spaces $H_{-k}^{E}$ and $H_{-k}^{A}$ for all $k \in \mathbb{N}$ may be found in the textbook by Engel and Nagel (2000).
Under the above assumptions, Eqns. 61) and 62 may be rewritten in the operator matrix form

$$
\left[\begin{array}{c}
\dot{x}(t) \\
\dot{z}(t)
\end{array}\right]=\left[\begin{array}{cc}
E & 0 \\
G C & A_{-1}
\end{array}\right]\left[\begin{array}{l}
x(t) \\
z(t)
\end{array}\right]+\left[\begin{array}{c}
B \\
H
\end{array}\right] u(t),
$$

where

$$
\left[\begin{array}{l}
x(0) \\
z(0)
\end{array}\right]=\left[\begin{array}{l}
x_{0} \\
z_{0}
\end{array}\right] \in H_{2}^{E} \times H^{A},
$$

and

$$
G C \in \mathscr{L}\left(H_{1}^{E}, H_{-1}^{A}\right)=\mathcal{H}^{\sim},
$$

and hence the equality (65) is understood in $H^{E} \times H_{-1}^{A}$ for every $t \geq 0$.

In the next step we introduce new state variables $[x(t) e(t)]^{T}$ defined by

$$
\left[\begin{array}{l}
x(t) \\
e(t)
\end{array}\right]=\left[\begin{array}{cc}
I & 0 \\
-M & I
\end{array}\right]\left[\begin{array}{l}
x(t) \\
z(t)
\end{array}\right], \quad t \geq 0
$$

where $M$ satisfies the condition $M \in \mathscr{L}\left(H^{E}, H^{A}\right)=\mathcal{H}$. This operator is to be chosen in such a way that the system operator matrix becomes diagonal, i.e.,

$$
\left[\begin{array}{c}
\dot{x}(t) \\
\dot{e}(t)
\end{array}\right]=\left[\begin{array}{cc}
E & 0 \\
0 & A_{-1}
\end{array}\right]\left[\begin{array}{l}
x(t) \\
e(t)
\end{array}\right]+\left[\begin{array}{c}
B \\
0
\end{array}\right] u(t),
$$

where

$$
\left[\begin{array}{l}
x(0) \\
e(0)
\end{array}\right]=\left[\begin{array}{c}
x_{0} \\
-M x_{0}+z_{0}
\end{array}\right] .
$$

One can check that the following holds:

$$
\begin{gathered}
{\left[\begin{array}{cc}
I & 0 \\
-M & I
\end{array}\right]\left[\begin{array}{cc}
I & 0 \\
M & I
\end{array}\right]=\left[\begin{array}{cc}
I & 0 \\
M & I
\end{array}\right]\left[\begin{array}{cc}
I & \\
-M & I
\end{array}\right]} \\
=\left[\begin{array}{ll}
I & 0 \\
0 & I
\end{array}\right]
\end{gathered}
$$

and hence the original state variables can be recovered by means of the expression

$$
\left[\begin{array}{l}
x(t) \\
z(t)
\end{array}\right]=\left[\begin{array}{cc}
I & 0 \\
M & I
\end{array}\right]\left[\begin{array}{l}
x(t) \\
e(t)
\end{array}\right], \quad t \geq 0 .
$$

In order to find a suitable operator $M \in \mathcal{H}$, we differentiate the relation (68), and then substitute (65) and (71). After simple manipulations we eventually obtain

$$
\begin{aligned}
& {\left[\begin{array}{c}
\dot{x}(t) \\
\dot{e}(t)
\end{array}\right]} \\
& =\left[\begin{array}{cc}
E & 0 \\
A_{-1} M-M E+G C & A_{-1}
\end{array}\right]\left[\begin{array}{l}
x(t) \\
e(t)
\end{array}\right] \\
& +\left[\begin{array}{c}
B \\
H-M B
\end{array}\right] u(t), \\
& {\left[\begin{array}{l}
x(0) \\
e(0)
\end{array}\right]=\left[\begin{array}{c}
x_{0} \\
-M x_{0}+z_{0}
\end{array}\right] \text {, }}
\end{aligned}
$$


which makes sense in $H^{E} \times H_{-1}^{A}$ for every $t \geq 0$. It is clear now that Eqn. (72) becomes (69) if the operator $M \in \mathcal{H}$ satisfies the following two operator equations:

$$
A_{-1} M h-M E h=-G C h, \quad h \in H_{1}^{E},
$$

where the equality is understood in $H_{-1}^{A}$, and

$$
H w=M B w, \quad w \in U,
$$

where the equality is understood in $H^{A}$. Since (73) has the form of the algebraic Sylvester equation (57) (with $E$ replaced by $-E$ ), we can use Corollary 2 to see that, if

$$
G C \in \mathcal{H}_{-1}
$$

(note that $\mathcal{H}_{-1}$ is now defined by means of the operators $A$ and $-E$ as opposite to $A$ and $E$ in the general setup of Sections 2 and 3 ) and

$$
\omega_{0}(T)+\omega_{0}(S)<0,
$$

then Eqn. (73) has a unique solution $M \in \mathcal{H}$ given by

$$
M=\int_{0}^{\infty} T_{-1}(t) G C S_{1}(-t) \mathrm{d} t .
$$

In the sequel we assume that the operators $A, G$ and $H$ satisfy the conditions (75), (76) and (74) (i.e., $H$ is given by $H=M B$ ).

It is easy to notice that under the above assumptions we can extend the class of solutions of the system 690 by assuming that $x_{0} \in H^{E}, z_{0} \in H^{A}$ and $u(\cdot) \in L_{\text {loc }}^{2}(0, \infty ; U)$. Understanding the solution of this system in the mild sense, we obtain

$$
\left[\begin{array}{c}
x(\cdot) \\
e(\cdot)
\end{array}\right] \in C\left([0, \infty) ; H^{E}\right) \times C\left([0, \infty) ; H^{A}\right),
$$

and hence, after taking (71) into account, it follows that also the mild solution of (65) for $x_{0} \in H^{E}, z_{0} \in H^{A}$ and $u(\cdot) \in L_{\mathrm{loc}}^{2}(0, \infty ; U)$ satisfies the condition

$$
\left[\begin{array}{l}
x(\cdot) \\
z(\cdot)
\end{array}\right] \in C\left([0, \infty) ; H^{E}\right) \times C\left([0, \infty) ; H^{A}\right) .
$$

We can now show that the dynamical system 62 may become an asymptotic state observer of the system (61). Since

$$
e(t)=z(t)-M x(t), \quad t \geq 0,
$$

and

$$
\|e(t)\|^{A}=\|T(t) e(0)\|^{A} \leq C_{1} e^{\omega_{1} t}\|e(0)\|^{A}, \quad t \geq 0,
$$

where $\omega_{1}$ is an arbitrary constant satisfying the condition $0>\omega_{1}>\omega_{0}(T)$, we get

$$
\lim _{t \rightarrow \infty}\|z(t)-M x(t)\|^{A}=0 .
$$

If, additionally, the operators $A$ and $G$ are such that $M \in$ $\mathcal{H}$ has a bounded inverse $M^{-1} \in \mathscr{L}\left(H^{A}, H^{E}\right)$, then for $t \geq 0$ we have $M^{-1} e(t)=M^{-1} z(t)-x(t)$ and the following condition holds:

$$
\lim _{t \rightarrow \infty}\left\|M^{-1} z(t)-x(t)\right\|^{E}=0 .
$$

This condition means that the system (62) is actually an asymptotic state observer for the control system 61). The rate of convergence (82) may be estimated by means of the inequality

$$
\begin{aligned}
& \left\|M^{-1} z(t)-x(t)\right\|^{E} \\
& \quad \leq\left\|M^{-1}\right\|_{\mathscr{L}\left(H^{A}, H^{E}\right)}\left\|z_{0}-M x_{0}\right\|^{A} C_{1} e^{\omega_{1} t}, \quad t \geq 0,
\end{aligned}
$$

and it follows that this convergence may be arbitrarily fast by a suitable choice of the growth bound $\omega_{0}(T)$ in the observer (61) (since $0>\omega_{1}>\omega_{0}(T)$ ).

In our considerations we have assumed that the composition of operators $G C \in \mathscr{L}\left(H_{1}^{E}, H_{-1}^{A}\right)=\mathcal{H}^{\sim}$ satisfies the stronger condition (75). It turns out that for this condition to be satisfied it is sufficient that $C \in$ $\mathscr{L}\left(H_{1}^{E}, Y\right)$ is an admissible output operator for the semigroup $(S(-t))_{t \geq 0} \subset \mathcal{H}^{E}$ and $G \in \mathscr{L}\left(Y, H_{-1}^{A}\right)$ is an admissible input operator for the semigroup $(T(t))_{t>0} \subset$ $\mathcal{H}^{A}$ (see Emirsajłow and Townley, 2000; 2005). These are very reasonable assumptions since in systems and control theories such conditions are widely explored. Moreover, these assumptions guarantee the well-posedness of the system $\{-E, B, C\}$ and the observer $\{A, H, G\}$.

In order to show that this is really the case, we prove the following lemma.

Lemma 3. Let the operator $F \in \mathcal{H}^{\sim}$ have the form

$$
F:=G K C \in \mathcal{H}^{\sim}
$$

where $C \in \mathscr{L}\left(H_{1}^{E}, Y\right), G \in \mathscr{L}\left(Y, H_{-1}^{A}\right), K \in \mathscr{L}(Y)$, If for some $t_{1}>0$ the operator $G \in \mathscr{L}\left(Y, H_{-1}^{A}\right)$ satisfies the condition

$$
\left\|\int_{0}^{t_{1}} T_{-1}\left(t_{1}-r\right) G w(r) \mathrm{d} r\right\|^{A} \leq b\left(t_{1}\right)\|w\|_{L^{2}\left(0, t_{1} ; Y\right)}
$$

for $w(\cdot) \in L^{2}\left(0, t_{1} ; Y\right)$, i.e., it is input admissible for the semigroup $(T(t))_{t \geq 0} \subset \mathcal{H}^{A}$, and the operator $C \in$ $\mathscr{L}\left(H_{1}^{E}, Y\right)$ satisfies the condition

$$
\left(\int_{0}^{t_{1}}\left\|C S_{1}\left(r-t_{1}\right) h\right\|_{Y}^{2} \mathrm{~d} r\right)^{1 / 2} \leq c\left(t_{1}\right)\|h\|^{E}
$$

for $h \in H_{1}^{E}$, i.e., it is output admissible for the semigroup $(S(-t))_{t \geq 0} \subset \mathcal{H}^{E}$, then for arbitrary $K \in \mathscr{L}(Y)$ the following holds:

$$
F=G K C \in \mathcal{H}_{-1}
$$


Proof. The assumptions (84) and (85) imply that

$$
\begin{aligned}
& \left\|\int_{0}^{t_{1}} \mathcal{U}^{\sim}\left(t_{1}-r\right) G K C \mathrm{~d} r h\right\|^{A} \\
& =\left\|\int_{0}^{t_{1}} T_{-1}\left(t_{1}-r\right) G K C S_{1}\left(r-t_{1}\right) h \mathrm{~d} r\right\|^{A} \\
& \leq b\left(t_{1}\right)\|K\|_{\mathscr{L}(Y)}\left(\int_{0}^{t_{1}}\left\|C S_{1}\left(r-t_{1}\right) h\right\|_{Y}^{2} \mathrm{~d} r\right)^{1 / 2} \\
& \leq b\left(t_{1}\right) c\left(t_{1}\right)\|K\|_{\mathscr{L}(Y)}\|h\|^{E}, \quad h \in H_{1}^{E},
\end{aligned}
$$

and hence the operator $((\mathcal{M} G K C)(t))_{t \geq 0} \subset \mathcal{H}^{\sim}$, defined by

$$
(\mathcal{M} G K C)(t):=\int_{0}^{t} \mathcal{U}^{\sim}(t-r) G K C \mathrm{~d} r, \quad t \geq 0
$$

enjoys certain properties. Namely, by extending the inequality 89 by continuity to all $h \in H^{E}$, we obtain

$$
(\mathcal{M} G K C)\left(t_{1}\right) \in \mathcal{H}
$$

and, since the functions $b(\cdot)$ and $c(\cdot)$ are non-decreasing in the interval $[0, \infty)$, we have

$$
\begin{aligned}
(\mathcal{M} G K C)(t) & \in \mathcal{H}, \quad t \in\left[0, t_{1}\right] \\
\sup _{0 \leq t \leq t_{1}}\|(\mathcal{M} G K C)(t)\| & \leq b\left(t_{1}\right) c\left(t_{1}\right)\|K\|_{\mathscr{L}(Y)}
\end{aligned}
$$

Next, by extending the inequality $(88)$ by continuity to all $h \in H^{E}$, we easily obtain the condition

$$
\tau-\lim _{t \searrow 0}(\mathcal{M} G K C)(t)=0 .
$$

According to Definition 4, the above three conditions mean that the operator $F=G K C \in \mathcal{H}^{\sim}$ is admissible for the Sylvester equation (40) and hence it satisfies the condition (86).

Finally, we point out that in order to guarantee the existence of a bounded inverse $M^{-1} \in \mathscr{L}\left(H^{A}, H^{E}\right)$ of the operator $M \in \mathscr{L}\left(H^{E}, H^{A}\right)$ one has to choose operators $A$ and $G$ with the following additional properties:

- There exists $\gamma>0$ such that

$$
\|M h\|^{A} \geq \gamma\|h\|^{E}, \quad h \in H^{E} .
$$

- The adjoint operator $M^{*} \in \mathscr{L}\left(H^{A}, H^{E}\right)$ satisfies

$$
\left\|M^{*} h\right\|^{E}>0, \quad(h \neq 0) \in H^{A} .
$$

The condition (91) guarantees that $M \in \mathscr{L}\left(H^{E}, H^{A}\right)$ is injective and has a closed range $\mathscr{R}(M)=\overline{\mathscr{R}(M)} \subset$ $H^{A}$. In turn, the condition 92 guarantees that the range $\mathscr{R}(M)$ is dense in $H^{A}$. Both these conditions together are equivalent to the fact that $M \in \mathscr{L}\left(H^{E}, H^{A}\right)$ is a bijection and consequently has a bounded inverse $M^{-1} \in$ $\mathscr{L}\left(H^{A}, H^{E}\right)$.

Using the explicit representation (77) of the operator $M$ we can rewrite (91) in the more explicit form

$$
\begin{gathered}
\left\|\int_{0}^{\infty} T_{-1}(t) G C S_{1}(-t) h \mathrm{~d} t\right\|^{A} \geq \gamma\|h\|^{E}, \\
h \in H_{1}^{E}
\end{gathered}
$$

and 91) in the form

$$
\begin{gathered}
\left\|\int_{0}^{\infty} S_{-1}^{*}(-t) C^{*} G^{*} T_{1}^{*}(t) h \mathrm{~d} t\right\|^{E}>0 \\
(h \neq 0) \in H_{1}^{A^{*}}
\end{gathered}
$$

where the meaning of the operators ${ }^{*}$ is rather clear.

It seems that examination of the conditions (91) and (92) for invertibility of the operator $M$ may be feasible if one works jointly with the Sylvester equation (73) and the above explicit conditions.

\section{Conclusion}

This paper provides a basic theory of infinite-dimensional Sylvester equations and develops a mathematical framework within which we can work out observers for a large class of infinite-dimensional control systems. It is clear that there are still problems to be studied. A difficult open problem it to guarantee the invertibility of the operator $M$. In order to achieve this invertibility, effective methods of how to choose the operators $A$ and $G$ of the observer have to be developed.

In general, the observer design problem based on the Sylvester equation (73) and Eqn. (74) has to be viewed as a problem where operators $E, B$ and $C$ are given and we are looking for operators $A, G$ and $M$ such that Eqns. (73) and (74) hold and the conditions (91) and 92) are satisfied.

On the other hand, infinite-dimensional Sylvester equations are of its own interests since they appear in several other control and systems theoretic problems. For some finite-dimensional examples we refer to the work of Gajic and Qureshi (2000). It is worth emphasizing that the famous Lyapunov equation is just a special case of the Sylvester equation. It seems that extending the concept of an implemented semigroup to the time-varying case may be a promising area of research.

For readers interested in the general mathematical theory which makes use of implemented semigroups in analysis of infinite-dimensional Sylvester and Lyapunov equations we recommend the book by Emirsajłow (2005). It also covers the problem of bounded perturbations in both of the Sylvester equations. 
Finally, it should be mentioned that the implemented semigroup is a special case of the so-called bi-continuous semigroup studied by Kuehnemund (2001). However, the level of generality in the discussion of Kuehnemund (2001) makes the results of little use from the control application point of view.

\section{References}

Alber, J. (2001). On implemented semigroups, Semigroup Forum 63(3): 371-386.

Arendt, W., Raebiger, F. and Sourour, A. (1994). Spectral properties of the operator equation $a x+x b=y$, Quarterly Journal of Mathematics 45(2): 133-149.

Chen, C.-T. (1984). Linear Systems Theory and Design, Holt, Rinehart and Winston, New York, NY.

Emirsajłow, Z. (2005). Implemented Semigroup in Analysis of Infinite-Dimensional Sylvester and Lyapunov equations, Technical University of Szczecin Press, Szczecin, (in Polish).

Emirsajłow, Z. and Townley, S. (2000). From PDEs with a boundary control to the abstract state equation with an unbounded input operator, European Journal of Control 7(1): 1-23.

Emirsajłow, Z. and Townley, S. (2005). On application of the implemented semigroup to a problem arising in optimal control, International Journal of Control 78(4): 298-310.

Engel, J. and Nagel, R. (2000). One-Parameter Semigroups for Linear Evolution Equations, Springer-Verlag, New York, NY.

Gajic, Z. and Qureshi, M. (2000). Lyapunov Matrix Equation in Systems Stability and Control, Academic Press, San Diego, CA.

Kuehnemund, F. (2001). Bi-continuous Semigroups on Spaces with Two Topologies: Theory and Applications, Ph.D. thesis, Eberhard-Karls-Univerität Tuebingen, Tuebingen.

Phong, V. Q. (1991). The operator equation $a x-x b=c$ with unbounded operators $a$ and $b$ and related Cauchy problems, Mathematische Zeitschrift 208(7): 567-588.

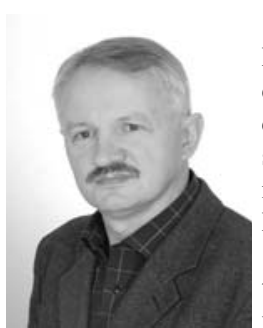

Zbigniew Emirsajłow was born in Warsaw, Poland, in 1954. He received his M.Sc. and Ph.D. degrees in electrical engineering from the Technical University of Szczecin in 1978 and 1983, respectively, and the D.Sc. degree in control engineering and robotics from the Systems Research Institute of the Polish Academy of Sciences in 1992. From 1978 to 2009 he was with the Institute of Control Engineering of the Technical University of Szczecin (1999-2002, 2005-2009: director of the Institute, 1993-2009: head of the Control Theory Group, 1994: university professor, 2007: full professor). In 1987 and 1992-93 he was appointed as a research fellow at the Control Theory Centre of the University of Warwick, and in 2004-2005 he was a visiting professor at the School of Engineering, Computer Science and Mathematics of the University of Exeter. Since 2009 he has been with the West Pomeranian University of Technology, Szczecin (head of the Control and Measurements Chair). He is the author and co-author of almost 90 journal articles and conference papers. His scientific interests cover mathematical control and systems theory, including infinite-dimensional control systems with unbounded input and output operators, infinite-dimensional Lyapunov, Sylvester and Riccati equations, as well as control of non-linear electromechanical systems.

\section{Appendix A}

\section{Proof of Lemma 1}

In order to prove (45), let us notice that every $t>0$ can be expressed in the form $t=N \sigma$, where $N \in \mathbb{N}, \sigma \in(0, \varepsilon)$ and $\varepsilon$ is the constant from Definition 4 . We have

$$
\begin{aligned}
& (\mathcal{M} F)(t) \\
& =\int_{0}^{N \sigma} \mathcal{U}^{\sim}(N \sigma-r) F \mathrm{~d} r \\
& =\sum_{k=1}^{N} \mathcal{U}^{\sim}((N-k) \sigma) \int_{(k-1) \sigma}^{k \sigma} \mathcal{U}^{\sim}(k \sigma-r) F \mathrm{~d} r \\
& =\sum_{k=1}^{N} \mathcal{U}^{\sim(N-k)}(\sigma) \int_{0}^{\sigma} \mathcal{U}^{\sim}(\sigma-r) F \mathrm{~d} r \\
& =\sum_{i=0}^{N-1} \mathcal{U}^{i}(\sigma)(\mathcal{M} F)(\sigma),
\end{aligned}
$$

which shows that $(\mathcal{M F})(t) \in \mathcal{H}$ and

$$
\begin{aligned}
\|(\mathcal{M} F)(t)\| & \leq\left(\sum_{i=0}^{N-1}\|\mathcal{U}(\sigma)\|_{\mathscr{L}(\mathcal{H})}^{i}\right)\|(\mathcal{M} F)(\sigma)\| \\
& =\frac{1-\|\mathcal{U}(\sigma)\|_{\mathscr{L}(\mathcal{H})}^{N}}{1-\|\mathcal{U}(\sigma)\|_{\mathscr{L}(\mathcal{H})}}\|(\mathcal{M} F)(\sigma)\|,
\end{aligned}
$$

where $\sum_{i=0}^{N-1}\|\mathcal{U}(\sigma)\|_{\mathscr{L}(\mathcal{H})}^{i}=N$ if $\|\mathcal{U}(\sigma)\|_{\mathscr{L}(\mathcal{H})}=1$

In turn, to prove (46) let us notice that for $t \geq 0$ and $\Delta \in(0, \varepsilon)$ we have

$$
\begin{aligned}
& (\mathcal{M F})(t+\Delta)-(\mathcal{M F})(t) \\
& =\int_{0}^{t+\Delta} \mathcal{U}^{\sim}(t+\Delta-r) F d r-\int_{0}^{t} \mathcal{U}^{\sim}(t-r) F d r \\
& =\left(\mathcal{U}^{\sim}(\Delta)-I\right) \int_{0}^{t} \mathcal{U}^{\sim}(t-r) F d r \\
& \quad+\int_{0}^{\Delta} \mathcal{U}^{\sim}(\Delta-r) F d r \\
& =(\mathcal{U}(\Delta)-I)(\mathcal{M} F)(t)+(\mathcal{M} F)(\Delta) .
\end{aligned}
$$

Since for $\Delta \searrow 0$ the right hand side of (93) is $\tau$ convergent to zero, we get

$$
\tau-\lim _{\Delta \searrow 0}((\mathcal{M} F)(t+\Delta)-(\mathcal{M} F)(t))=0,
$$

which proves the continuity from the right. Furthermore, 
for $t>0$ and $\Delta>0$ such that $t-\Delta>0$ we have

$$
\begin{aligned}
& (\mathcal{M} F)(t)-(\mathcal{M F})(t-\Delta) \\
& =\int_{0}^{t} \mathcal{U}^{\sim}(t-r) F \mathrm{~d} r-\int_{0}^{t-\Delta} \mathcal{U}^{\sim}(t-\Delta-r) F \mathrm{~d} r \\
& =\int_{0}^{\Delta} \mathcal{U}^{\sim}(t-r) F \mathrm{~d} r \\
& =\mathcal{U}^{\sim}(t-\Delta) \int_{0}^{\Delta} \mathcal{U}^{\sim}(\Delta-r) F \mathrm{~d} r \\
& =\mathcal{U}(t-\Delta)((\mathcal{M} F)(\Delta)) \\
& =T(t-\Delta)((\mathcal{M} F)(\Delta)) S(t-\Delta) .
\end{aligned}
$$

Since for every $h \in X^{E}$ we obtain

$$
\begin{aligned}
& p_{h}((\mathcal{M F} F)(t)-(\mathcal{M} F)(t-\Delta)) \\
&=\|T(t-\Delta)((\mathcal{M} F)(\Delta)) S(t-\Delta) h\|^{A} \\
&= \| T(t-\Delta)((\mathcal{M} F)(\Delta))(S(t-\Delta)-S(t)) h \\
&+T(t-\Delta)((\mathcal{M} F)(\Delta)) S(t) h \|^{A} \\
& \leq\|T(t-\Delta)\|_{\mathcal{H}^{A}}(\|(\mathcal{M} F)(\Delta)\| \|(S(t-\Delta) \\
&\left.-S(t)) h\left\|^{E}+\right\|((\mathcal{M} F)(\Delta)) S(t) h \|^{A}\right),
\end{aligned}
$$

where $(T(t))_{t \geq 0} \subset \mathcal{H}^{A}$ is locally uniformly bounded and $((\mathcal{M F})(t))_{t \geq 0} \subset \mathcal{H}$ is uniformly bounded in the neighbourhood of origin, we get

$$
\tau-\lim _{\Delta \searrow 0}((\mathcal{M} F)(t)-(\mathcal{M} F)(t-\Delta))=0 .
$$

This proves the continuity from the left and thus and (96) together imply (46).

\section{Appendix B}

\section{Proof of Lemma 2}

Let $(\cdot)^{\prime}$ denote $\tau^{\sim}$-differentiation and let all integrals be understood as Riemann $\tau^{\sim}$-integrals, i.e., integrals convergent in $\left(\mathcal{H}^{\sim}, \tau^{\sim}\right)$. Using integration by parts, we easily obtain that

$$
\begin{aligned}
& \int_{0}^{t} \mathcal{U}^{\sim}(t-r) F \mathrm{~d} r \\
& \quad=\int_{0}^{t} \mathcal{U}^{\sim}(t-r)(r F)^{\prime} \mathrm{d} r \\
& =t F+\mathcal{A}^{\sim} \int_{0}^{t} r \mathcal{U}^{\sim}(t-r) F \mathrm{~d} r, \quad t \geq 0
\end{aligned}
$$

Using integration by parts once again, we also obtain that

$$
\begin{aligned}
\int_{0}^{t} r \mathcal{U}^{\sim}(t-r) F \mathrm{~d} r \\
=\int_{0}^{t} r\left(\int_{0}^{r} \mathcal{U}^{\sim}(t-s) F \mathrm{~d} s\right)^{\prime} \mathrm{d} r \\
=t \int_{0}^{t} \mathcal{U}^{\sim}(t-r) F \mathrm{~d} r \\
\quad-\int_{0}^{t} \mathcal{U}^{\sim}(t-r)\left(\int_{0}^{r} \mathcal{U}^{\sim}(r-s) F \mathrm{~d} s\right) \mathrm{d} r
\end{aligned}
$$

where $t \geq 0$. Since both the terms on the right-hand side of (98) take values in $\mathscr{D}\left(\mathcal{A}^{\sim}\right)=\mathcal{H}_{1}^{\sim}$, we can substitute (98) into 977 and obtain Lemma 2 .

Received: 6 February 2011 Revised: 24 August 2011 